Published in final edited form as:

Curr Mol Med. 2009 March ; 9(2): 94-99.

\title{
Pathogenesis of Central Nervous System Tuberculosis
}

\author{
Nicholas A. Be ${ }^{1}$, Kwang Sik Kim², William R. Bishai ${ }^{1}$, and Sanjay K. Jain ${ }^{*}, 1,2$ \\ ${ }^{1}$ Center for Tuberculosis Research, Johns Hopkins University School of Medicine, Baltimore, \\ Maryland, USA \\ 2Division of Pediatric Infectious Diseases, Johns Hopkins University School of Medicine, \\ Baltimore, Maryland, USA
}

\begin{abstract}
Central Nervous System (CNS) tuberculosis is a serious, often fatal form of tuberculosis, predominantly affecting young children. HIV co-infection and drug resistant strains of Mycobacterium tuberculosis are making the diagnosis and treatment of CNS tuberculosis more complicated. Current concepts about the pathogenesis of CNS tuberculosis are based on necropsy studies done in 1933, which suggest that tuberculous meningitis develops subsequent to the rupture into the cerebrospinal fluid of tuberculomas that form around M. tuberculosis deposited in the brain parenchyma and meninges during the initial hematogenous dissemination.

Foreign antigens including pathogens deposited in the brain parenchyma are not detected efficiently by the immune system in the CNS. These experimental data may explain the clinical observation of delayed "paradoxical" enlargement or development of intracranial tuberculomas, observed several weeks to months in patients receiving anti-tuberculous therapy.

Since severe sequelae are observed even when CNS tuberculosis is treated effectively, it is important to develop preventive strategies for this disease. Recent data utilizing animal models suggests that, in addition to host factors, M. tuberculosis genes and their encoded proteins may contribute specifically to bacterial invasion and survival in the CNS. Understanding how these microbial factors affect CNS disease would be essential to developing such preventive strategies.
\end{abstract}

In spite of the decline of tuberculosis (TB) incidence within industrialized nations over the course of recent decades, this disease remains prominent worldwide and is still one of the leading causes of infection-related mortality across the world. According to the World Health Organization, there are approximately 9.2 million new TB cases and 1.7 million deaths every year [1]. Central nervous system (CNS) TB is a serious, often fatal disease predominantly affecting young children [2-4]. CNS TB is difficult to diagnose and treat; treatment includes four drugs, developed more than 30 years ago, and they only prevent death or disability in less than half of patients. M. tuberculosis resistant to these drugs threatens a return to an era of even greater mortality [5]. Two major forms of CNS tuberculosis include meningitis which accounts for $0.5-1 \%$ of tuberculous disease and intracranial tuberculomas which on a global level account for up to $40 \%$ of 'brain tumors' [3, 6].

*Address correspondence to this author at the Center for Tuberculosis Research, 1550 Orleans Street, Baltimore, MD 21231, USA; Tel: 410-614-8241; Fax: 410-614-8173; sjain5@jhmi.edu. 
Multi-drug resistant (MDR) Mycobacterium tuberculosis are on the rise, and approximately $4.8 \%$ of new TB cases worldwide are due to MDR strains, representing 489,139 patients annually [7]. Treatment of MDR-TB especially in the HIV co-infected individuals is much more complex than in the case of fully drug-susceptible organisms, and is associated with higher treatment cost and longer treatment periods [8]. In addition, such cases exhibit poorer patient outcome and higher mortality rates $[8,9]$. MDR-TB meningitis is especially challenging to treat due to limited CNS penetration of several $2^{\text {nd }}$ line anti-TB drugs. Nonspecific clinical presentation, especially in young children, poor diagnostics, and delays in instituting appropriate anti-TB therapy, as drug susceptibility testing may require up to 10 weeks, further increase the risk of mortality or the onset of severe, irreversible neurological damage $[10,11]$.

TB is the most common human immunodeficiency virus (HIV)-related opportunistic infection and the most important cause of morbidity and mortality in HIV-infected individuals in the developing world [12]. Dual infection with HIV and M. tuberculosis was responsible for 600,000 deaths in 2004 [13]. Of the 2 billion latently infected with $M$. tuberculosis, many develop reactivation disease, years after the initial exposure. Coinfection with HIV increases the risk of development of reactivation tuberculosis disease from a lifetime risk of 5-10\% to approximately $10 \%$ per year [14-16]. Unfortunately, the number of dual infections with HIV and M. tuberculosis is increasing at an alarming rate with 2 million new double infections in 2004 alone [13]. Co-infection with HIV not only increases the risk of development of CNS tuberculosis several times, [17] but may also lead to a much higher case-fatality rate [18].

\section{PATHOGENESIS OF CNS TB AND SUBSEQUENT TB MENINGITIS}

The initial point of tuberculosis infection is entry of the bacilli into the lungs via inhalation of infectious droplets, whereupon the bacteria colonize macrophages within the alveoli. During the progression of active pulmonary disease, bacteria may disseminate to local lymph nodes and bloodstream, whereupon spread throughout the systemic circulatory system may occur. It is also likely that extensive bacteremia following dissemination from the lungs increases the probability that a sub-cortical focus will be established in the CNS [19]. Therefore, higher numbers of bacilli in the circulatory system may be associated with increased likelihood of CNS invasion and subsequent CNS TB.

The CNS is protected from the systemic circulatory system by the physiological blood brain barrier (BBB). This barrier is principally composed of tightly apposed human brain microvascular endothelial cells (Fig. 1). The basal portion of these endothelial cells is supported by astrocyte processes interspersed with the extracellular matrix [20]. Paracellular transport is limited by the presence of endothelial cell tight junctions, while transcellular movement is restricted by the relative paucity of endocytic vesicles. Such properties render the barrier impermeable to many large, hydrophilic molecules and circulating pathogens. Also protective of the CNS is the blood- cerebrospinal fluid (CSF) barrier, providing spatial separation of the circulatory system from the CSF at the choroid plexus. Cells lining the blood-CSF barrier share similar properties to those lining the BBB, with enhanced tight junctions and more stringent regulation of transcytosis. Despite the integrity of this barrier, 
however, there are a number of bacterial and viral pathogens capable of crossing the BBB and causing subsequent meningitis / encephalitis.

Much of the current understanding of the pathogenesis of CNS TB and subsequent meningitis comes from the meticulous work of Arnold Rich and Howard McCordock, who demonstrated upon autopsy that the majority of TB meningitis patients displayed a caseating focus in the brain parenchyma or the meninges. Rich postulated that these foci, also termed as "Rich foci," develop around bacteria deposited in the meninges and brain parenchyma during the initial bacteremic phase. Much later, the rupture of these foci allowed dissemination of the bacilli into the subarachnoid space, causing diffuse, inflammatory meningitis [21] (Fig. 2). Since the meninges and the brain parenchyma are anatomically and physiologically protected from the systemic circulation by the BBB, the mechanism(s) by which the bacilli initially invade this barrier need to be elucidated. Theoretically, $M$. tuberculosis can cross the BBB as a free (extra-cellular) organism or via infected monocytes/neutrophils. While the latter hypothesis seems attractive, such cellular traffic is severely restricted into the CNS prior to invasion by the offending pathogen [22]. Intravenous inoculation of free M. tuberculosis or M. bovis in guinea pigs and rabbits has been shown to produce CNS invasion as evidenced by the formation of tuberculomas in their brain parenchyma [21]. Further, one report utilizing CD18-/- leukocyte adhesion deficient mice, suggests that free mycobacteria may traverse the BBB independent of leukocytes or macrophages [23]. Finally, it is unclear whether, after invading the CNS, $M$. tuberculosis reside primarily within the parenchyma of the brain, the vessel wall, or the endothelial cells lining the microvasculature. Significant vasculitis associated with CNS tuberculosis [24] and robust human endothelial cell invasion observed in vitro [25] may suggest that M. tuberculosis reside, at least initially, in the endothelial cells lining the microvasculature.

The spread of M. tuberculosis into the subarachnoid space following rupture of a Rich focus triggers a robust inflammatory $\mathrm{T}$ cell response. Studies of CSF cytokine levels in patients with TB meningitis have found elevated levels of TNF- $\alpha$ and IFN- $\gamma$ [26]. The clinical manifestation of CNS tuberculosis is primarily a consequence of the inflammation which develops in response to M. tuberculosis in the CNS. Obstruction of the CSF by inflammatory infiltrate leads to hydrocephalus, and vasculitis contributes to infarction, causing potentially irreparable neurological damage. Inhibition of this inflammation may therefore help in preventing the sequelae of CNS TB. Though thalidomide, which inhibits TNF-a, has not be shown to be beneficial for the treatment of TB meningitis in children [27], corticosteroids such as dexamethasone which suppress the production of inflammatory cytokines and chemokines lead to better outcomes and are recommended as adjunctive treatment for patients with TB meningitis [28, 29].

\section{SELECTIVE AND MODIFIED IMMUNE REACTIVITY IN THE CNS}

The brain parenchyma exists within a unique microenvironment, distinct in a number of different ways. Though commonly referred to as "immunologically privileged", immune reactivity in the brain parenchyma can be better referred as being selective and modified [22, 30], meaning that immune response to the presence of foreign antigen is relatively limited. 
Such a characteristic is essential in order to limit inflammatory damage within an organ as indispensable and minimally regenerative as the brain. Selective and modified immune reactivity within the parenchyma is mediated by a number of different cellular and physiological factors. Antigen presentation within the parenchyma is severely limited, as the presence of dendritic cells is highly restricted and there is low level of expression of MHC class II molecules in the intact human brain [30]. In addition, microglia, the macrophages of the brain, exhibit relatively poor antigen presenting capabilities and tend to induce apoptosis of effector cells rather than proliferation [31]. Access of systemic lymphocytes to the parenchyma is limited by both the blood brain barrier [22] as well as soluble and cell surface factors. Neuron-dependent conversion of T-cells into FoxP3+ T-regulatory cells that suppress inflammation is instrumental in regulating CNS inflammation [32].

Selective / modified immune reactivity within the brain parenchyma may explain why inflammatory meningitis is not established immediately following disseminated TB. Rich and McCordock observed that inflammatory meningitis often occurred many months after the incidence of active disease [21]. Studies have shown that there is an absence of T-cell or antibody response to PPD testing following intracranial injection of heat-killed Bacillus Calmette-Guérin (BCG) in rats, indicating that BCG escapes immune recognition within the parenchyma [33]. Studies have also shown that subsequent peripheral sensitization of the immune system results in the development of an immune-mediated delayed-type hypersensitivity (DTH) response, and inflammatory lesions surrounding the heat killed BCG within the CNS [34]. These experimental data are in concordance with the clinical observation of delayed "paradoxical" intracranial tuberculomas, which develop in patients several weeks to months following anti-TB therapy [6]. Further, it should be noted that though the brain parenchyma has limited immune surveillance, the CSF has a much more robust immune response to foreign antigens [22]. This is consistent with the observation that rupture of the "Rich foci" into CSF containing subarachnoid space causes diffuse, inflammatory meningitis.

The idea that M. tuberculosis can hide in the CNS for months to years is intriguing. A review of 100 autopsies of patients with late generalized tuberculosis performed between 1937-1959, revealed that 54\% of patients had evidence of meningitis and caseous CNS foci that may have been the probable sources for hematogenous dissemination [35]. Though late onset TB meningitis may occur in adults and older children, in young children, meningitis is likely to be concurrent with miliary TB, as part of primary tuberculosis [19] (Fig. 3).

\section{MODELS FOR THE STUDY OF CNS TB}

\section{Models of Mycobacterial Translocation Across In Vitro Cellular Barriers}

Although the majority of research on in vitro infections by $M$. tuberculosis involves the invasion of macrophages by the bacilli, similar interactions with non-professional antigen presenting cells can be equally informative to understand the pathogenesis of myco bacterial disease. Several investigators have studied the interaction of $M$. tuberculosis with cell types that form cellular barriers [36, 37]. Bermudez et al. have shown that $M$. tuberculosis invade A549 pulmonary epithelial cells with an efficiency of 2 to $3 \%$ of the initial inoculum. Interestingly, though bacteria did not invade the endothelial cells efficiently, bacteria 
passaged through A549 cells were subsequently able to be taken up by endothelial cells with an efficiency of 5 to $6 \%$ of the inoculum. In addition, free as well as intracellular (within infected monocytes) $M$. tuberculosis were able to traverse a bi-cellular monolayer (epithelial and endothelial cells) efficiently [37]. A study by Menozzi et al. has shown that the 28-kDa heparin-binding haemagglutinin adhesin (HBHA), required for extrapulmonary dissemination of the bacilli [38], induces a reorganization of the actin filament network in confluent endothelial cells, but does not affect the tight junctions that link them. When coupled to colloidal gold particles, HBHA-mediates attachment of the particles to the membrane of HEp-2 epithelial cells and A549 epithelial cells. After attachment, the particles are internalized in membrane-bound vacuoles that migrate across A549 cells to reach the basal side [39]. These observations suggest that $M$. tuberculosis HBHA induces receptormediated endocytosis and epithelial transcytosis, which may represent a macrophageindependent extra-pulmonary dissemination mechanism leading to systemic infection by $M$. tuberculosis.

An in vitro model composed of a monolayer of primary human brain microvascular endothelial cells (HBMEC) was used to study bacterial invasion and translocation across the BBB by M. tuberculosis [25]. It was shown that M. tuberculosis strains H37Rv and CDC1551 are capable of invading and traversing the BBB with much higher efficiency than the non-pathogenic M. smegmatis by an active actin-dependent process. By microarray profiling and experiments with selected $M$. tuberculosis transposon mutants, this study also suggested that specific M. tuberculosis genes may be required for this invasion and traversal.

Though relatively inexpensive and easy to use, in vitro models such as the ones described above have limitations. This is because in vitro systems utilize one or more cells lines grown in one or more compartments, and therefore cannot mimic the complex interactions of the several cell types and compartments representing a living in vivo system. Nonetheless, these models may prove to be extremely useful as screening and proof of principle tools.

\section{In Vivo Models of CNS Invasion}

Past studies have indicated that genotypic differences between strains of M. tuberculosis can contribute to variations in virulence, host immune response, and clinical outcome of disease due to differences in their cellular and molecular profiles [40-44]. The existence of bacterial genetic determinants of invasion is also supported by limited clinical data. In a study looking at extra-pulmonary tuberculosis during co-infection with multiple $M$. tuberculosis strains, it was observed that only one of the infecting strains was capable of establishing an extrapulmonary infection, suggesting clonal selection during the process of dissemination [45]. Data from one study has also shown that there was strain specific compartmentalization of M. tuberculosis in the lung or the CNS [46]. However, despite the above findings, a study performed on patients from the Western Cape Province, South Africa failed to identify an association between $M$. tuberculosis genotype and patient presentation or clinical outcome [47].

Gilla Kaplan and colleagues have developed a rabbit model for experimental TB meningitis, where rabbits are infected via intra-cisternal injection of $M$. bovis Ravenel [43, 48]. An acute inflammatory response in the CNS is induced by high dose infection $\left(10^{7}\right.$ colony forming 
units), or a delayed, chronic infection is induced by employing a lower inoculum. Studies in the rabbit model have also shown that intracisternal infection by the HN878 or W4 strains of M. tuberculosis result in higher bacillary loads, persistent elevation of TNF-a within the CSF, and more severe clinical symptoms [43]. van Well et al. have developed a similar model in mice, where mice were infected intra-cerebrally with high-dose of M. tuberculosis H37Rv [49]. All infected mice developed meningitis, with extensive cellular infiltrate in the CNS and meningeal inflammation. Though the intra-cisternal / intra-cerebral injection models may prove to be useful tools for studying acute or chronic inflammatory tuberculous meningitis, a major limitation of these models is that they do not simulate natural CNS disease, caused by hematogenous dissemination of $M$. tuberculosis and subsequent invasion of the CNS. For the same reasons, these models cannot be used to understand the mechanism(s) by which the bacteria initially gain access to the CNS.

Our laboratory has focused on developing models to study invasion of the CNS by $M$. tuberculosis and simulate natural human CNS TB as part of hematogenously disseminated disease. Rich and McCordock challenged both sensitized and non-sensitized guinea pigs and rabbits intravenously in their seminal work on TB meningitis [21]. M. bovis were used for infecting rabbits, while $M$. tuberculosis $\mathrm{H} 37 \mathrm{Rv}$ were used for guinea pigs. Though all animals developed few to several granulomatous lesions in the brain parenchyma and meninges, none developed acute exudative meningitis. These data suggest that unlike other meningitis causing bacteria, $M$. tuberculosis do not enter the CSF directly through the bloodCSF barrier at the level of choroid plexi. Instead, they enter the brain parenchyma or meninges at the level of the BBB.

Based on these studies done by Rich et al. we opted to detect $M$. tuberculosis in the whole brain tissue, including the parenchyma, instead of just the CSF. Our data indicate that $M$. tuberculosis are detected in the brain tissue at each time point after intravenous infection [50]. Further, no significant immune response, as measured by histopathological and cytokine profile analysis, was elicited in the infected brain tissue, compared with extensive inflammation noted in the infected lung tissue at the same time. This model was subsequently used to study the role of $M$. tuberculosis genes in the pathogenesis of CNS disease. A pool of defined transposon disruption mutants of M. tuberculosis CDC1551 was used to infect mice intravenously. The study identified specific $M$. tuberculosis mutants (representing M. tuberculosis genes Rv0311, Rv0805, Rv0931c, Rv0986 and MT3280) which displayed CNS-specific phenotypes that were absent in lung tissue [50]. It is hoped that more extensive use of this model will likely identify further genetic determinants for CNS invasion and/or survival by M. tuberculosis, and will bring to light potential targets for treatment of the disease.

In summary, despite a century and a quarter after the discovery of $M$. tuberculosis, remarkably little is known about the pathogenesis of CNS TB. Since severe sequelae are observed even when CNS TB is treated effectively, it is important to develop preventive strategies for this disease. Understanding how M. tuberculosis invade and survive initially in the CNS would be essential to developing such preventive strategies. Future studies on the genetic determinants of $M$. tuberculosis and their interactions with the blood brain barrier 
will hopefully lead to a more extensive understanding of CNS TB / TB meningitis and ways in which it may be combated.

\section{REFERENCES}

[1]. WHO Report 2008: Global tuberculosis control-surveillance, planning, financing. Vol. 2008. World Health Organization; 2008. p. 1-294.

[2]. Lincoln EM, Sordillo VR, Davies PA. J. Pediatr. 1960; 57:807-823. [PubMed: 13762229]

[3]. Jacobs, RF.; Starke, JR. Mycobacterium tuberculosis. In: Long, SS.; Pickering, LK.; Prober, CG., editors. Principles and practice of pediatric infectious diseases. Churchill Livingstone; New York, NY: 2003.

[4]. Girgis NI, Sultan Y, Farid Z, Mansour MM, Erian MW, Hanna LS, Mateczun AJ. Am. J. Trop. Med. Hyg. 1998; 58:28-34. [PubMed: 9452288]

[5]. Thwaites GE, Hien TT. Lancet Neurol. 2005; 4:160-170. [PubMed: 15721826]

[6]. Jain SK, Kwon P, Moss WJ. Clin. Pediatr. (Phila). 2005; 44:443-450. [PubMed: 15965552]

[7]. WHO Report 2008: Anti-tuberculosis drug resistance in the world. 2008:1-120. Report No. 4.

[8]. Wells CD, Cegielski JP, Nelson LJ, Laserson KF, Holtz TH, et al. J. Infect. Dis. 2007196:S86S107. [PubMed: 17624830]

[9]. Gandhi NR, Moll A, Sturm AW, Pawinski R, Govender T, Lalloo U, Zeller K, Andrews J, Friedland G. Lancet. 2006; 368:1575-1580. [PubMed: 17084757]

[10]. Sofia M, Maniscalco M, Honore N, Molino A, Mormile M, et al. Int. J. Tuberc. Lung Dis. 2001; 5:551-558. [PubMed: 11409583]

[11]. Padayatchi N, Bamber S, Dawood H, Bobat R. Pediatr. Infect. Dis. J. 2006; 25:147-150. [PubMed: 16462292]

[12]. Harries, A.; Maher, D.; Graham, S. 2nd. World Health Organization; 2004. p. 1-210.

[13]. Kaufmann SH, McMichael AJ. Nat. Med. 2005; 11:S33-44. [PubMed: 15812488]

[14]. Stead WW. Am. Rev. Respir. Dis. 1967; 95:729-745. [PubMed: 4960690]

[15]. Selwyn PA, Hartel D, Lewis VA, Schoenbaum EE, Vermund SH, Klein RS, Walker AT, Friedland GH. N. Engl. J. Med. 1989; 320:545-550. [PubMed: 2915665]

[16]. Daley CL, Small PM, Schecter GF, Schoolnik GK, McAdam RA, Jacobs WR Jr. Hopewell PC. N. Engl. J. Med. 1992; 326:231-235. [PubMed: 1345800]

[17]. Berenguer J, Moreno S, Laguna F, Vicente T, Adrados M, Ortega A, Gonzalez-LaHoz J, Bouza E. N. Engl. J. Med. 1992; 326:668-672. [PubMed: 1346547]

[18]. Katrak SM, Shembalkar PK, Bijwe SR, Bhandarkar LD. J. Neurol. Sci. 2000; 181:118-126. [PubMed: 11099721]

[19]. Donald PR, Schaaf HS, Schoeman JF. J. Infect. 2005; 50:193-195. [PubMed: 15780412]

[20]. Rubin LL, Staddon JM. Annu. Rev. Neurosci. 1999; 22:11-28. [PubMed: 10202530]

[21]. Rich AR, McCordock HA. Bull. Johns Hopkins Hosp. 1933; 52:5-37.

[22]. Ransohoff RM, Kivisakk P, Kidd G. Nat. Rev. Immunol. 2003; 3:569-581. [PubMed: 12876559]

[23]. Wu HS, Kolonoski P, Chang YY, Bermudez LE. Infect. Immun. 2000; 68:2979-2984. [PubMed: 10768998]

[24]. Thwaites GE, Chau TT, Drobniewski F, McAdam K. J. Neurol. Neurosurg. Psychiatry. 2000; 68:289-299. N.T., M. al., e. [PubMed: 10675209]

[25]. Jain SK, Paul-Satyaseela M, Lamichhane G, Kim KS, Bishai WR. J. Infect. Dis. 2006; 193:12871295. [PubMed: 16586367]

[26]. Mastroianni CM, Paoletti F, Lichtner M, D'Agostino C, Vullo V, Delia S. Clin. Immunol. Immunopathol. 1997; 84:171-176. [PubMed: 9245549]

[27]. Schoeman JF, Springer P, van Rensburg AJ, Nurs D, Swanevelder S, Hanekom WA, Haslett PA, Kaplan G. J. Child. Neurol. 2004; 19:250-257. [PubMed: 15163089]

[28]. Rock RB, Hu S, Gekker G, Sheng WS, May B, Kapur V, Peterson PK. J. Infect. Dis. 2005; 192:2054-2058. [PubMed: 16288367] 
[29]. Thwaites GE, Nguyen DB, Nguyen HD, Hoang TQ, Do TT, Nguyen TC, Nguyen HQ, Nguyen TT, Nguyen NH, Nguyen TN, Nguyen NL, Nguyen HD, Vu NT, Cao HH, Tran TH, Pham PM, Nguyen TD, Stepniewska K, White NJ, Tran TH, Farrar JJ. N. Engl. J. Med. 2004; 351:17411751. [PubMed: 15496623]

[30]. Galea I, Bechmann I, Perry VH. Trends Immunol. 2007; 28:12-18. [PubMed: 17129764]

[31]. Ford AL, Foulcher E, Lemckert FA, Sedgwick JD. J. Exp. Med. 1996; 184:1737-1745. [PubMed: 8920862]

[32]. Liu Y, Teige I, Birnir B, Issazadeh-Navikas S. Nat. Med. 2006; 12:518-525. [PubMed: 16633347]

[33]. Matyszak MK, Perry VH. J. Neuroimmunol. 1998; 82:73-80. [PubMed: 9526848]

[34]. Matyszak MK, Perry VH. Neuroscience. 1995; 64:967-977. [PubMed: 7753389]

[35]. Slavin RE, Walsh TJ, Pollack AD. Medicine (Baltimore). 1980; 59:352-366. [PubMed: 7432152]

[36]. Birkness KA, Deslauriers M, Bartlett JH, White EH, King CH, Quinn FD. Infect. Immun. 1999; 67:653-658. [PubMed: 9916072]

[37]. Bermudez LE, Sangari FJ, Kolonoski P, Petrofsky M, Goodman J. Infect. Immun. 2002; 70:140146. [PubMed: 11748175]

[38]. Pethe K, Alonso S, Biet F, Delogu G, Brennan MJ, Locht C, Menozzi FD. Nature. 2001; 412:190-194. [PubMed: 11449276]

[39]. Menozzi FD, Reddy VM, Cayet D, Raze D, Debrie AS, Dehouck MP, Cecchelli R, Locht C. Microbes Infect. 2005:1-9. [PubMed: 15914062]

[40]. Lopez B, Aguilar D, Orozco H, Burger M, Espitia C, Ritacco V, Barrera L, Kremer K, Hernandez-Pando R, Huygen K, van Soolingen D. Clin. Exp. Immunol. 2003; 133:30-37. [PubMed: 12823275]

[41]. Theus SA, Cave MD, Eisenach KD. J. Infect. Dis. 2005; 191:453-460. [PubMed: 15633105]

[42]. McShane H. Clin. Exp. Immunol. 2003; 133:20-21. [PubMed: 12823273]

[43]. Tsenova L, Ellison E, Harbacheuski R, Moreira AL, Kurepina N, Reed MB, Mathema B, Barry CE 3rd, Kaplan G. J. Infect. Dis. 2005; 192:98-106. [PubMed: 15942899]

[44]. Gagneux S, Small PM. Lancet Infect. Dis. 2007; 7:328-337. [PubMed: 17448936]

[45]. Garcia de Viedma D, Marin M, Ruiz Serrano MJ, Alcala L, Bouza E. J. Infect. Dis. 2003; 187:695-699. [PubMed: 12599090]

[46]. Garcia de Viedma D, Marin M, Andres S, Lorenzo G, Ruiz-Serrano MJ, Bouza E. Pediatr. Infect. Dis. J. 2006; 25:457-459. [PubMed: 16645515]

[47]. Maree F, Hesseling AC, Schaaf HS, Marais BJ, Beyers N, van Helden P, Warren RM, Schoeman JF. Pediatr. Infect. Dis. J. 2007; 26:13-18. [PubMed: 17195699]

[48]. Tsenova L, Sokol K, Freedman VH, Kaplan G. J. Infect. Dis. 1998; 177:1563-1572. [PubMed: 9607834]

[49]. Van Well GTJ, Wieland CW, Florquin S, Roord JJ, van der Poll T, van Furth AM. J. Infect. Dis. 2007; 195:694-697. [PubMed: 17262711]

[50]. Be NA, Lamichhane G, Grosset J, Tyagi S, Cheng Q, Kim KS, Bishai WR, Jain SK. J. Infect. Dis. 2008; 198:1520-1528. [PubMed: 18956986] 


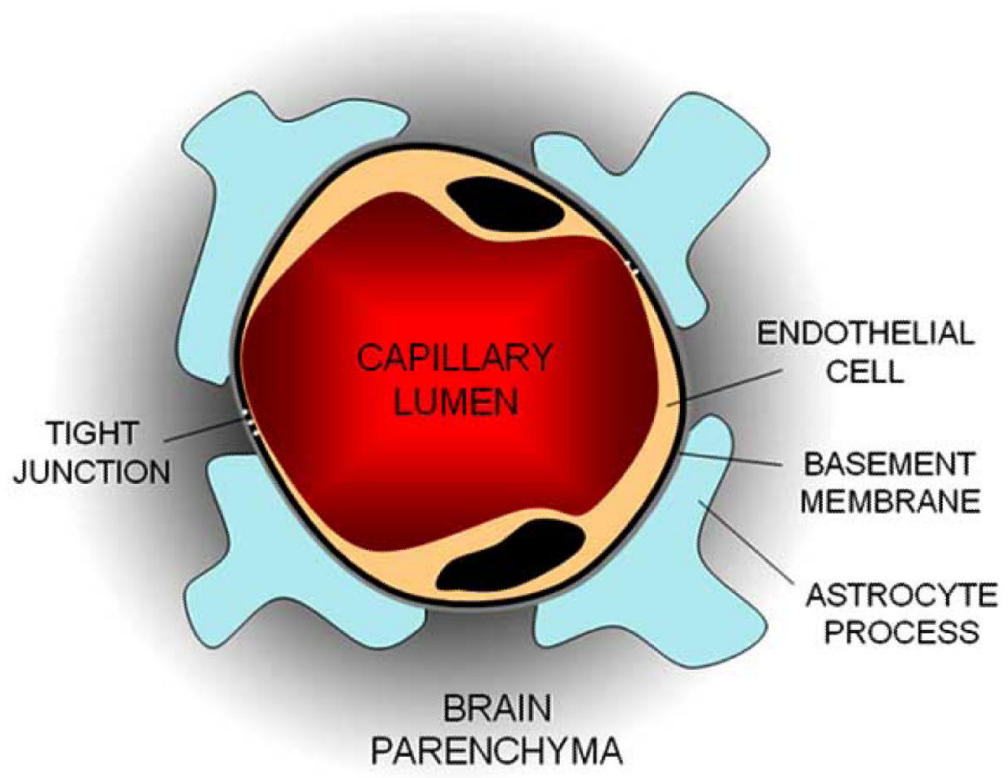

Fig. (1).

The blood brain barrier is composed primarily of brain microvascular endothelial cells, the basal portions of which are supported by astrocyte processes. These endothelial cells afford protection of the central nervous system (CNS) by limiting access between the CNS and the systemic circulatory system. Such limited access is facilitated by specialized tight junctions and minimal transcytosis, thereby greatly reducing paracellular and transcellular movement. 


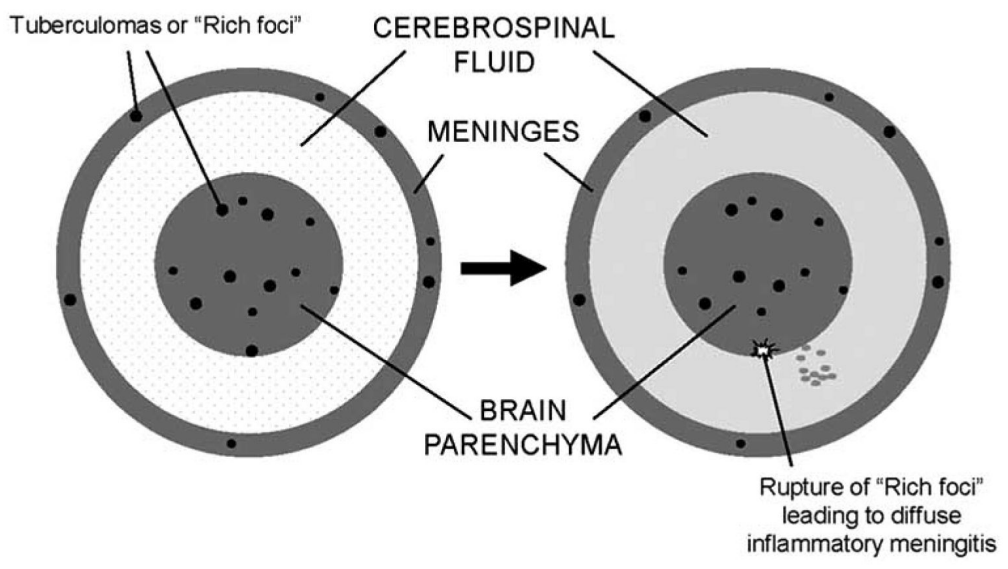

Fig. (2). Pathogenesis of Central Nervous System tuberculosis and subsequent tuberculous meningitis

In their seminal publication on Central Nervous System (CNS) tuberculosis, Arnold Rich and Howard McCordock demonstrated upon autopsy that the majority of tuberculous meningitis patients displayed a caseating focus in the brain parenchyma or the meninges. They postulated that during primary infection, $M$. tuberculosis get deposited in the brain parenchyma and meninges during hematogenous dissemination. Rich and McCordock challenged both sensitized and non-sensitized guinea pigs and rabbits intravenously with $M$. tuberculosis and $M$. bovis respectively. Though no animal developed acute exudative meningitis, all animals developed few to several granulomatous lesions in the brain parenchyma and meninges. Based on these data, Rich further postulated that tuberculomas or "Rich foci" develop around the deposited mycobacteria. Much later, the rupture of these foci allows dissemination of mycobacteria into the cerebrospinal fluid, causing diffuse, inflammatory meningitis. 


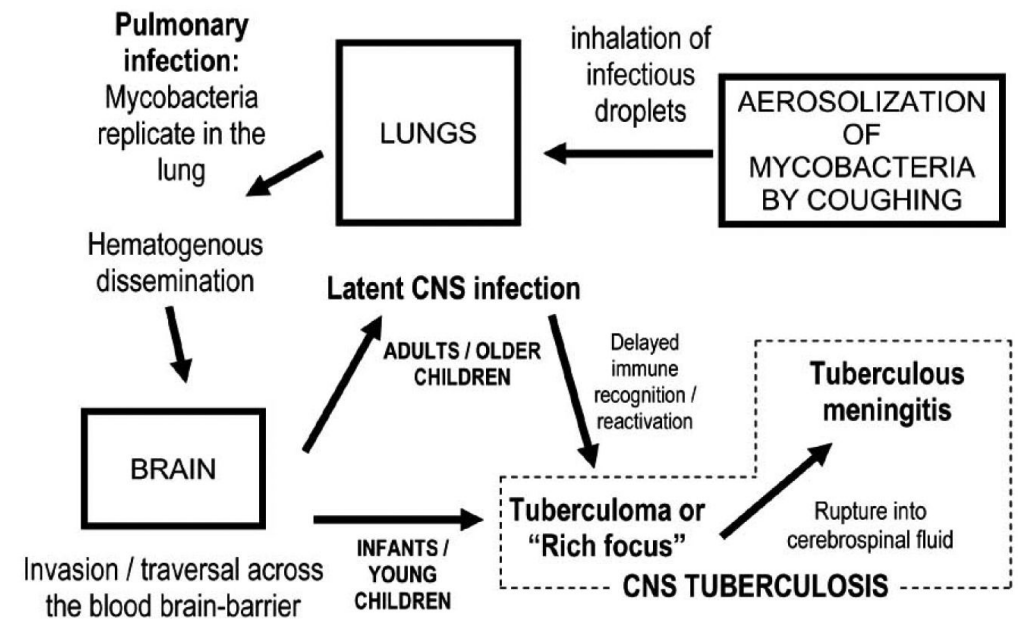

Fig. (3). Tuberculosis: Spread to the Central Nervous System

The establishment of Central Nervous System (CNS) tuberculosis occurs after hematogenous dissemination of mycobacteria from the lungs. The mycobacteria invade / traverse the blood brain barrier and get deposited in the CNS. When deposited in large numbers as part of primary tuberculosis in infants / young children, they may cause tuberculosis meningitis and formation of tuberculomas. However, in adults / older children, deposited mycobacteria may not elicit any immune response and cause latent disease until immune recognition / reactivation causes formation of tuberculomas in the CNS. These tuberculomas may later rupture into the cerebrospinal fluid, leading to severe inflammation and tuberculous meningitis. 